\title{
Biomarker Positive Malignant Neoplasm
}

National Cancer Institute

\section{Source}

National Cancer Institute. Biomarker Positive Malignant Neoplasm. NCI Thesaurus. Code C150123.

A finding indicating the presence of a biomarker in a tumor sample from a patient with a malignant neoplasm. 